Draft VERSiON JANUARY 8, 2018

Typeset using $\mathrm{LAT}_{\mathrm{E}} \mathrm{X}$ twocolumn style in AASTeX61

\title{
GENERAL-RELATIVISTIC SIMULATIONS OF FOUR STATES OF ACCRETION ONTO MILLISECOND PULSARS
}

\author{
${ }^{1}$ Lawrence Berkeley National Laboratory, 1 Cyclotron Road, Berkeley, CA 94720 \\ ${ }^{2}$ Departments of Physics and Astronomy, and Theoretical Astrophysics Center, UC Berkeley, CA 94720 \\ ${ }^{3}$ Center for Interdisciplinary Exploration 8 Research in Astrophysics (CIERA), Physics 8 Astronomy, Northwestern University, Evanston, \\ IL 60202 \\ ${ }^{4}$ Kavli Institute for Theoretical Physics, Kohn Hall, University of California at Santa Barbara, Santa Barbara, CA 93106
}

\begin{abstract}
Accreting neutron stars can power a wide range of astrophysical phenomena including short- and long-duration gamma-ray bursts, ultra-luminous X-ray sources, and X-ray binaries. Numerical simulations are a valuable tool for studying the accretion-disk-magnetosphere interaction that is central to these problems, most clearly for the recently discovered transitional millisecond pulsars. However, magnetohydrodynamic (MHD) methods, widely used for simulating accretion, have difficulty in highly magnetized stellar magnetospheres, while force-free methods, suitable for such regions, cannot include the accreting gas. We present an MHD method that can stably evolve essentially forcefree, highly magnetized regions, and describe the first time-dependent relativistic simulations of magnetized accretion onto millisecond pulsars. Our axisymmetric general-relativistic MHD simulations for the first time demonstrate how the interaction of a turbulent accretion flow with a pulsar's electromagnetic wind can lead to the transition of an isolated pulsar to the accreting state. This transition naturally leads to the formation of relativistic jets, whose power can greatly exceed the power of the isolated pulsar's wind. If the accretion rate is below a critical value, the pulsar instead expels the accretion stream. More generally, our simulations produce for the first time the four possible accretion regimes, in order of decreasing mass accretion rate: (a) crushed magnetosphere and direct accretion; (b) magnetically channeled accretion onto the stellar poles; (c) the propeller state, where material enters through the light cylinder but is prevented from accreting by the centrifugal barrier; (d) almost perfect exclusion of the accretion flow from the light cylinder by the pulsar wind.
\end{abstract}

Keywords: pulsars: general — stars: neutron - X-rays: binaries — accretion, accretion disks — magnetohydrodynamics (MHD) - relativistic processes

kparfrey@lbl.gov

* Einstein Fellow

$\dagger$ Theoretical Astrophysics Center Fellow 


\section{INTRODUCTION}

Accretion onto rotating neutron stars is the principal agent underlying several classes of high-energy objects, including nuclear- and accretion-powered millisecond Xray pulsars (AMXPs; van der Klis 2000; Patruno \& Watts 2012), the atoll sources and the rapidly accreting $\mathrm{Z}$ sources (Hasinger \& van der Klis 1989). Two particularly interesting varieties were recently discovered: the transitional millisecond pulsars, which switch between X-ray and radio pulsing states (Papitto et al. 2013; Stappers et al. 2014), and the highly super-Eddington pulsing ultra-luminous X-ray sources (ULXs; Bachetti et al. 2014; Fürst et al. 2016; Israel et al. 2017). Making sense of these objects requires understanding how turbulent, magnetized accretion flows interact with stellar magnetospheres.

Non-relativistic MHD simulations have been extensively employed to study accretion onto magnetized stars, displaying plasmoid ejections, accretion columns, magnetically powered jets, and centrifugal inhibition of accretion (e.g. Hayashi et al. 1996; Miller \& Stone 1997; Romanova et al. 2002; Kato et al. 2004; Zanni \& Ferreira 2009; Romanova et al. 2012; Lii et al. 2014). Simulations of this kind become more challenging in the relativistic regime, because of the need to maintain nearly forcefree (highly magnetically dominated) regions alongside the accreting plasma. Relativistic MHD simulations of isolated-pulsar magnetospheres have been performed by modifying the evolution of the densities and velocities (Komissarov 2006; Tchekhovskoy, Spitkovsky, \& Li 2013). On the other hand, force-free electrodynamics, a simplified form of high-magnetization MHD, has been used to study the interaction of pulsar magnetospheres with accretion disks, which were kinematically specified rather than dynamically evolved (Parfrey, Spitkovsky, \& Beloborodov 2017). Here we describe a new approach to seamlessly combine in one simulation a full-MHD accretion disk interacting with an essentially force-free stellar magnetosphere.

\section{NUMERICAL APPROACH}

We carry out the simulations in spherical coordinates $(r, \theta, \phi)$ with a modified version of the HARMPI $\operatorname{code}^{1}$, which is based on HARM2D (Gammie et al. 2003; Noble et al. 2006). We evolve the ideal GRMHD equations,

$$
\begin{aligned}
\nabla_{\mu}\left(\rho u^{\mu}\right) & =0 \\
\nabla_{\mu} T_{\nu}^{\mu} & =0, \\
\nabla_{\mu} F^{* \mu \nu} & =0,
\end{aligned}
$$

\footnotetext{
${ }^{1}$ https://github.com/atchekho/harmpi
}

where

$T^{\mu \nu}=\left(\rho+\frac{\epsilon+p}{c^{2}}+\frac{b^{2}}{4 \pi c^{2}}\right) u^{\mu} u^{\nu}+\left(p+\frac{b^{2}}{8 \pi}\right) g^{\mu \nu}-\frac{b^{\mu} b^{\nu}}{4 \pi}$

is the total energy-momentum tensor; $\rho, \epsilon$, and $p$ are the fluid-frame mass density, internal-energy density, and gas pressure; $u^{\mu}$ is the fluid four-velocity; $b^{\mu}$ is the magnetic four-vector $\left(b^{2} / 8 \pi\right.$ is the fluid-frame magnetic pressure); and $F^{* \mu \nu}=b^{\mu} u^{\nu}-b^{\nu} u^{\mu}$ is the dual of the electromagnetic field tensor. Vector components written as $V_{\hat{\alpha}}$ have the same dimensions as the physical quantity they represent, and are defined as $V_{\hat{\phi}}=\operatorname{sign}\left(V^{\phi}\right) \sqrt{V_{\phi} V^{\phi}}$.

After the solution is stepped forward in time according to Eqn. (1), we adjust the hydrodynamic variables $\rho, \epsilon$, and $u^{\mu}$ inside the force-free magnetosphere for improved behavior in the high-magnetization regime.

To distinguish the force-free magnetosphere from the accretion flow, we introduce a new variable $\mathcal{F}$ which is evolved as a passive scalar, $\nabla_{\mu}\left(\mathcal{F} \rho u^{\mu}\right)=0 . \mathcal{F}$ is the fraction of a given zone's mass density that can be identified as magnetospheric, or due to the density "floor" which is required for numerical stability: $\mathcal{F}=1$ in the isolated-pulsar magnetosphere before the onset of accretion, and $\mathcal{F} \approx 0$ inside the accretion flow. Additionally, we specify a smooth, fixed radial profile $\mathcal{P}(r)$, whose purpose is to decrease the severity of the adjustments with increasing $r$, such that they vanish beyond the light cylinder: $\mathcal{P}=1$ at the stellar surface, $r=r_{*}$, and $\mathcal{P}=0$ for $r \geq R_{\mathrm{LC}}$, where $R_{\mathrm{LC}}=c / \Omega$ is the lightcylinder (LC) radius and $\Omega$ is the stellar spin angular frequency. We make all adjustments according to the value of $\mathcal{A}=1-\mathcal{F P}$.

When $\mathcal{A}=1$ (i.e., inside the accretion flow or in any cell beyond the light cylinder) the evolution is unmodified and follows the GRMHD equations. When $\mathcal{A}=0$ the mass and internal-energy densities are set to fixed background distributions, and the velocity parallel to the magnetic field, as measured by the coordinate-static observer, ${ }^{2}$ is set to zero. For $0<\mathcal{A}<1$ the modification to these quantities interpolates linearly between these two extremes. When mass or internal energy is added to (or removed from) a cell, the fluid velocity is adjusted, such that the total conserved momentum parallel to the magnetic field, as measured by the coordinate-static observer, is unchanged.

At the inner radial boundary, $r=r_{*}$, we extrapolate the mass and internal-energy densities into the star. We fix the radial magnetic field to its dipolar distribution, and extrapolate the tangential components. For surface

\footnotetext{
${ }^{2}$ Coordinate-static observer: $n^{\alpha}=\left(n^{t}, 0^{i}\right)$
} 
locations at which accretion occurs, we set the velocities to corotation with the prescribed stellar angular velocity $\Omega$, plus the extrapolated value of the velocity along the magnetic field, as measured by the corotating observer. ${ }^{3}$ For non-accreting locations we set the velocity such that the coordinate-static observer measures it to be the component of the stellar angular velocity that is perpendicular to the magnetic field.

We have confirmed that in flat spacetime, using our boundary conditions and treatment of magnetically dominated regions, we reproduce the analytic solution for a rotating monopole (Michel 1973) and the flux distribution and spin-down power of a rotating dipole (Contopoulos et al. 1999; Gruzinov 2005). The numerical methods and associated tests will be described in detail in a future paper.

\section{PROBLEM CONFIGURATION}

We initialize the problem with an equilibrium hydrodynamic torus (Chakrabarti 1985; De Villiers et al. 2003), with the inner edge at $r=60 r_{\mathrm{g}}$, where $r_{\mathrm{g}}=$ $G M / c^{2}$ is the gravitational radius, density maximum $\rho_{\max }=1$ at $r_{\max }=85 r_{\mathrm{g}}$, and angular momentum distribution $\propto r^{1 / 3}$. We insert a magnetic field loop into the torus, with plasma $\beta=8 \pi p / b^{2} \geq 100$ and poloidal field lines following density isosurfaces, $A_{\phi} \propto \rho$, and add random, 10\%-level pressure variations to jump-start the magnetorotational instability (MRI; Balbus \& Hawley 1991). We consider two orientations of the torus magnetic field: parallel or anti-parallel to the stellar dipole field at the equator.

The magnetosphere, occupying the entire computational domain outside the torus, is dominated by the neutron star's magnetic field. We initialize it using the vector potential of a static, potential dipole of magnetic moment $\mu$ in the Schwarzschild spacetime (Wasserman \& Shapiro 1983), measured in units of $\mu_{\text {unit }}=$ $r_{\mathrm{g}}^{3} \sqrt{4 \pi \rho_{\max } c^{2}}$. For rotating stars, we deform the stellar field lines, which would be rotationally opened by an isolated pulsar, to go around and close beyond the torus. This prevents these field lines from becoming artificially trapped by the torus when the star begins to rotate.

Near the star the gas and internal-energy densities create a hydrostatic atmosphere, with zero velocity relative to the coordinates, that smoothly transitions to $\rho, \epsilon \propto$ $r^{-6}$ and $\epsilon / \rho=0.035$ at $r \gtrsim R_{\mathrm{LC}}$. The magnetosphere is highly magnetically dominated, with the initial magnetization near the star $\sigma=b^{2} / 4 \pi \rho c^{2} \sim 3 \times 10^{4} \gg 1$.

We performed 19 simulations in two sets. In the first, $\mu=[1.25,2.5,5,10,20,40,80,160], R_{\mathrm{LC}}=20 r_{\mathrm{g}}$,

\footnotetext{
${ }^{3}$ Corotating observer: $n^{\alpha}=n^{t}(1,0,0, \Omega)$
}

for both orientations of the torus magnetic field. In the second, $\mu=20, R_{\mathrm{LC}} / r_{\mathrm{g}}=[20,40,60, \infty]$, for the antiparallel stellar-torus field orientation. We ran the simulations for $\Delta t=5 \times 10^{4} \mathrm{r}_{\mathrm{g}} / c$, or $\sim 10$ Keplerian orbits at $r_{\text {max }}$.

We set the stellar radius to $r_{*}=4 r_{\mathrm{g}}$; this choice for typical $r_{*}=10 \mathrm{~km}$ gives the spin frequency $\nu \approx$ $950\left(R_{\mathrm{LC}} / 20 r_{\mathrm{g}}\right)^{-1} \mathrm{~Hz}$. We use the Kerr spacetime in the Boyer-Lindquist foliation, with spin parameter $a=$ $(1 / 3)\left(R_{\mathrm{LC}} / 20 r_{\mathrm{g}}\right)^{-1}$ (e.g. Belyaev \& Parfrey 2016); the results are essentially unchanged when we instead use the Kerr-Schild spacetime foliation.

The numerical resolution is $N_{r} \times N_{\theta} \times N_{\varphi}=$ $1024 \times 512 \times 1$ in the domain extending to $r_{\text {out }}=$ $5 \times 10^{3} r_{\mathrm{g}}$. The radial grid is logarithmically spaced for $r \leq 200 r_{\mathrm{g}}$, beyond which it becomes hyperexponential (Tchekhovskoy et al. 2009); the angular grid is mildly concentrated toward the midplane. We use Lax-Friedrichs fluxes with the monotonized-central limiter.

\section{RESULTS}

The isolated-pulsar magnetosphere is established on an Alfvén-crossing timescale (e.g. Contopoulos et al. 1999; Spitkovsky 2006; Parfrey et al. 2012). Rotating open field lines form a pulsar wind that engulfs the torus, well before the MRI begins to destroy the torus's equilibrium at $t \approx 3,000 r_{\mathrm{g}} / c$. For our simulation with $\mu=5, R_{\mathrm{LC}}=20 r_{\mathrm{g}}$, and the anti-parallel orientation of stellar dipole and torus magnetic fields, this results in an accretion disk that flows inward against the pulsar wind, and into the LC. The disk's magnetic field is free to reconnect with the star's closed field due to their anti-parallel orientation, placing the disk's gas on the stellar field lines. The newly reconnected field line may be added to the pulsar's wind of open magnetic flux, centrifugally ejecting the threaded gas. Otherwise, the stellar field channels the disk material into accretion columns, which form at the magnetospheric radius $R_{\mathrm{m}}$ at which the stellar field truncates the equatorial flow, as seen in Fig. 1(a). Matter enters the columns with low radial velocity and is gravitationally accelerated to $v_{\hat{r}} \approx-c / 3$ at the stellar surface. Our boundary conditions allow the matter to pass undisturbed through the surface, so no accretion shock forms.

Figure 1(b) shows the smooth regions of 'swept back' toroidal magnetic field ${ }^{4}$ connected to the stellar poles that form the pulsar wind, spinning down the star. Matter in the accretion columns along the edge of the closed

\footnotetext{
${ }^{4}$ Measured by the observer normal to spatial hypersurfaces: $n_{\alpha}=\left(n_{t}, 0_{i}\right)$.
} 

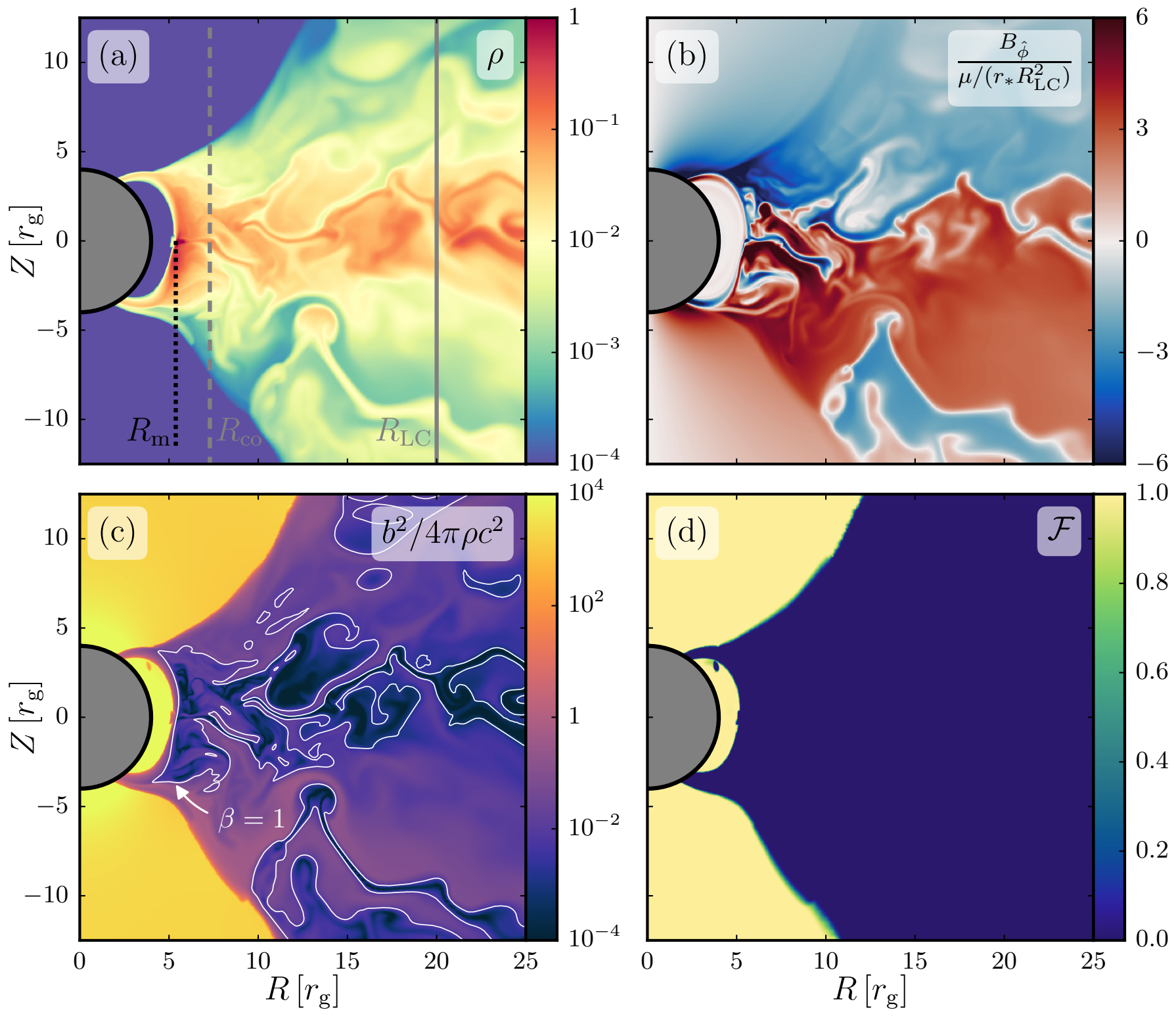

Figure 1. Quasi-steady magnetically channeled accretion; the $\mu=5, R_{\mathrm{LC}}=20 r_{\mathrm{g}}$, anti-parallel magnetic field simulation at $t=16,000 r_{\mathrm{g}} / c$. (a) Mass density; vertical lines indicate the corotation (gray dashed), light-cylinder (gray solid), and magnetospheric (black dotted) radii. (b) Toroidal magnetic field. (c) Magnetization $\sigma$, with $\beta=1$ surfaces in white. (d) Passively advected scalar $\mathcal{F}$, the magnetospheric mass-density fraction.

zone initially rotates faster than the star and pulls the stellar field forward, generating $B_{\hat{\phi}}$ of opposite sign to that in the wind and delivering a spin-up torque. The irregularity in $B_{\hat{\phi}}$ in the disk reflects MHD turbulence down to $R_{\mathrm{m}}$.

The columns are magnetically dominated $(\sigma>1)$ at their bases; elsewhere the accretion flow is matterdominated [Fig. 1(c)]. Near the magnetospheric boundary, the accretion flow's pressure is largely thermal $(\beta>1)$, while $\beta<1$ in the columns, the mass-loaded wind, and in some of the equatorial regions. The magne- tospheric regions are strongly magnetically dominated, with $\sigma>10^{4}$ near the star, enabled by our method of combining force-free and standard-GRMHD evolution. The passive scalar $\mathcal{F}$ is shown in Fig. $1(\mathrm{~d}): \mathcal{F} \approx 0$ indicates plasma from the torus, while the $\mathcal{F} \approx 1$ region is magnetospheric; the absence of plasma with intermediate $\mathcal{F} \sim 0.5$ supports this means of distinguishing between force-free and MHD regions.

The location of the magnetospheric radius is determined by the balance between the magnetic pressure in the closed zone and the hydrodynamic pressure in the 


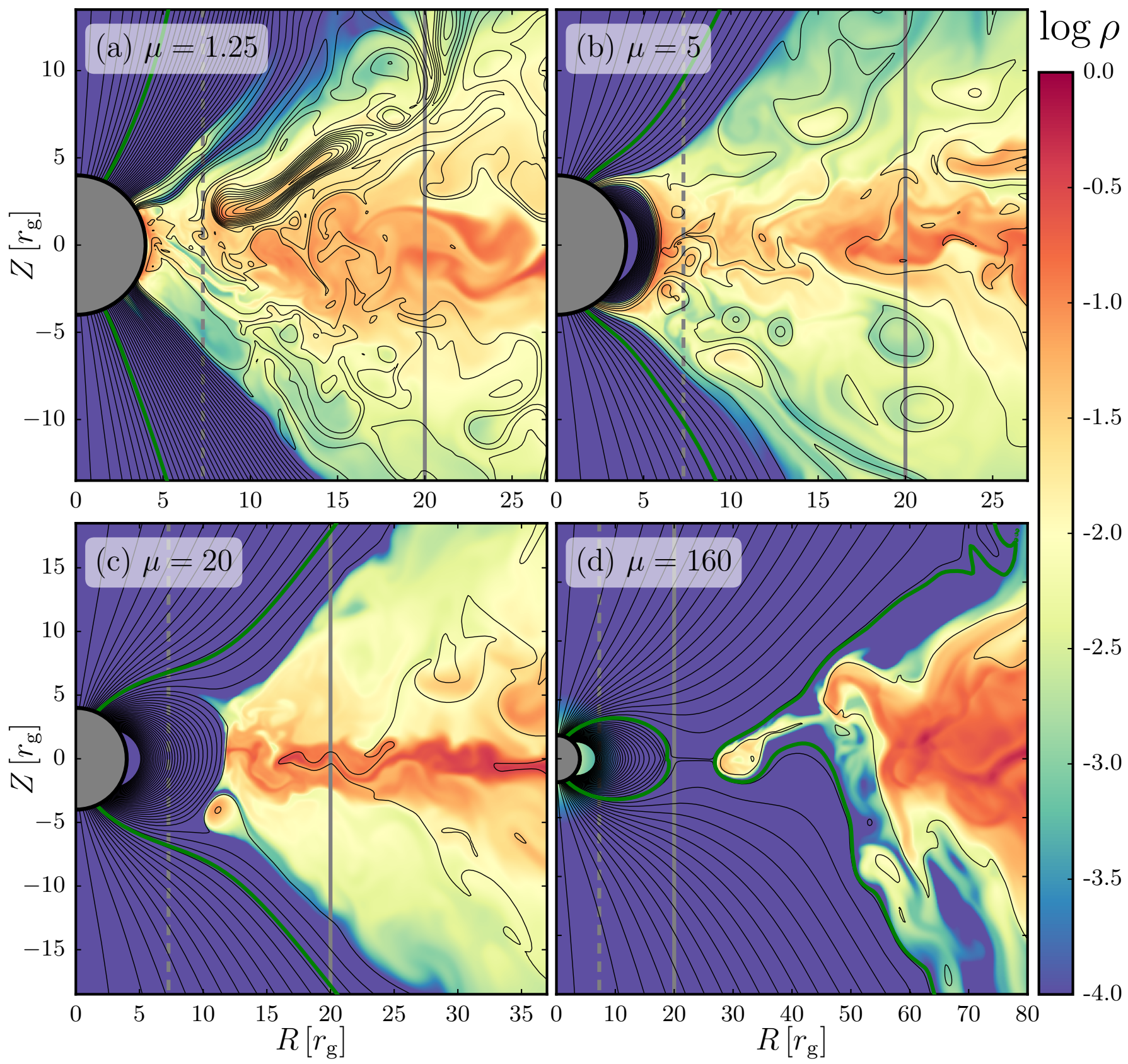

Figure 2. Four accretion states, determined by the stellar magnetic moment $\mu$ for the same initial torus and stellar spin frequency. Poloidal magnetic field lines are in black, equispaced in magnetic flux, with the thicker green line representing the last closed field line for an equivalent isolated pulsar. Gray vertical lines indicate $R_{\mathrm{co}}$ and $R_{\mathrm{LC}}$, as in Fig. 1. (a) Direct accretion. (b) Magnetically channeled accretion onto the stellar poles. (c) The propeller state. (d) Exclusion of the accretion flow from the light cylinder, shown immediately following the clearing of the inner magnetosphere.

inner accretion flow. By varying the star's magnetic moment $\mu$, while leaving its rotation rate and the initial gas torus unchanged, ${ }^{5}$ we can move $R_{\mathrm{m}}$ in or out, produc-

\footnotetext{
${ }^{5}$ Equivalent to fixing $\mu$ and varying the torus density, and hence the accretion rate.
}

ing distinct states of the combined magnetosphere-disk system.

Figure 2 illustrates these states (stellar and torus magnetic fields are initially anti-parallel). Whenever the accreting plasma enters the LC there is more open magnetic flux than in the isolated case, leading to a stronger electromagnetic pulsar wind (Parfrey et al. 
2016). This wind is collimated by the accretion flow, forming Poynting-flux-dominated relativistic jets.

At the lowest magnetic moment (equivalently, highest accretion rate), almost all of the star's closed field lines are either opened or crushed, leading to direct accretion near the equator [Figure 2(a)]. The flow does not spread across the stellar surface due to our infall boundary conditions. At larger $\mu$ the star's magnetic field truncates the disk, with accretion proceeding via magnetically confined columns [Figure 2(b)].

At yet higher $\mu$, a state similar to the classical propeller regime is formed [Figure 2(c)] in which the accretion flow is truncated between the corotation radius $R_{\mathrm{co}}=\left(R_{\mathrm{LC}}-a r_{\mathrm{g}}\right)^{2 / 3} r_{\mathrm{g}}^{1 / 3}$ and $R_{\mathrm{LC}}$. The accretion rate is very low in this state, with only rare instances of lowdensity streams reaching the stellar surface. Matter is centrifugally ejected from near $R_{\mathrm{m}}$ when reconnection attaches it to the rotating magnetosphere. An intermediate state, with $R_{\mathrm{m}} \approx R_{\mathrm{co}}$, is also possible [see Figure $3(\mathrm{a})]$.

At the highest magnetic moment, the pulsar wind is powerful enough to almost entirely exclude the accretion flow from the LC. In the simulation shown in Figure 2(d) the flow enters the LC four times, each time only residing for $\approx 900 r_{\mathrm{g}} / c$, or roughly seven stellar spin periods, before being entirely thrown back out again. None of the gas reaches the stellar surface.

We find another intermediate state $(\mu=80)$ in which the accretion flow spends most of the simulation inside the LC, but is occasionally ejected. When inside the LC the flow is strongly churned by interaction with the stellar field.

Our initial conditions, in which the stellar field lines are deformed around, and do not penetrate, the torus lying beyond $R_{\mathrm{LC}}$, allow a clean test of the fieldorientation effect (Figure 3). When the direction of the torus's poloidal magnetic loop is reversed, the initial field strength $|\boldsymbol{B}|$ remains unchanged everywhere in the domain. Figure 3(a) shows that, for anti-parallel fields, reconnection between the stellar and disk fields permits the opening of originally closed field lines and the formation of long-lived accretion columns. This reconnection does not occur in the parallel case, and the accreting plasma instead pushes the closed field lines inward (e.g. Romanova et al. 2011). Rather than opening the stellar field, the accretion flow increases the amount of closed flux, Figure 3(b). Stable columns are not formed, and accretion is more time-dependent, as plasma diffuses onto the stellar field in the region of large gradients at $R_{\mathrm{m}}$.

In Figure 4 we show global quantities as a function of stellar magnetic moment $\mu$, time-averaged between $t=$
20,000-50,000 $r_{\mathrm{g}} / c$; vertical lines indicate the standard deviation, and hence the variability.

The magnetospheric radius $R_{\mathrm{m}}$ increases with increasing $\mu$, and is similar for the two field orientations at all $\mu$, as seen instantaneously in Figure 3. The scaling resembles the standard $R_{\mathrm{m}} \propto \mu^{4 / 7}$ dependence (e.g. Pringle \& Rees 1972).

The mass accretion rate is very low or zero when the disk is held beyond corotation $(\mu \geq 20)$, and is otherwise approximately independent of $\mu$. The rate is higher with anti-parallel fields, as stable accretion columns form and gas can fall onto the star without diffusing across field lines. The MRI does not create a self-sustaining dynamo in these axisymmetric simulations, and so the accretion is transient, appearing as a long outburst; $\dot{M}$ therefore has a large variability. The other quantities of interest remain relatively steady.

The amount of force-free magnetic flux through the $r=R_{\mathrm{LC}}$ surface, $\psi_{\mathrm{open}}$, varies strongly as $\mu$ decreases and $R_{\mathrm{m}}$ moves inward, with $\psi_{\text {open }}$ increasing over its isolated-pulsar value for anti-parallel fields and decreasing to nearly zero in the parallel case (see also Figure 3). The relativistic jet power (flux of electromagnetic energy through $r=R_{\mathrm{LC}}$, in the force-free region) roughly follows the open flux as $L_{\text {jet }} \propto \psi_{\text {open }}^{2}$. Similarly, the total stellar torque $N_{\text {tot }}$ becomes increasingly negative (i.e., gives more rapid spin-down) as accretion increases the open magnetic flux (anti-parallel fields) and retreats toward zero torque for the closed-magnetosphere solutions (parallel fields). Here the open flux, torque, and jet power are normalized by their values in the absence of accretion; note $L_{0}=-N_{0} \Omega=\mu^{2} \Omega^{4} / c^{3}$ (Gruzinov 2005). See Parfrey et al. (2016) for further discussion on using these isolated-pulsar values to create dimensionless variables.

The shaded regions in Figure 4 show the range predicted by a simple model, in which the spin-down torque and jet power are related to the magnetic flux opened by the accretion flow, estimated via $\psi_{\text {open }}=$ $\zeta\left(R_{\mathrm{LC}} / R_{\mathrm{m}}\right) \psi_{\text {open,0}}$, where $\zeta$ is an efficiency parameter (Parfrey et al. 2016, 2017). The jet power is then $L_{\text {jet }}=\zeta^{2}\left(R_{\mathrm{LC}} / R_{\mathrm{m}}\right)^{2} L_{0}$. The figure suggests that opening can be efficient, with $\zeta>0.5$ for anti-parallel fields.

Figure 5 illustrates the dependence of the global quantities on the stellar spin frequency $\Omega(\mu=20$ in all cases). The magnetospheric radius is insensitive to $\Omega$. $\dot{M}$ onto the star depends weakly on $\Omega$ when accretion occurs, and is zero for the propeller system. The open magnetic flux is above its isolated-pulsar value in all cases, leading to an enhancement in the power of the electromagnetic wind or jet, which is a strong function of the stellar spin frequency, $L_{\text {jet }} \propto \Omega^{3.6}$. 

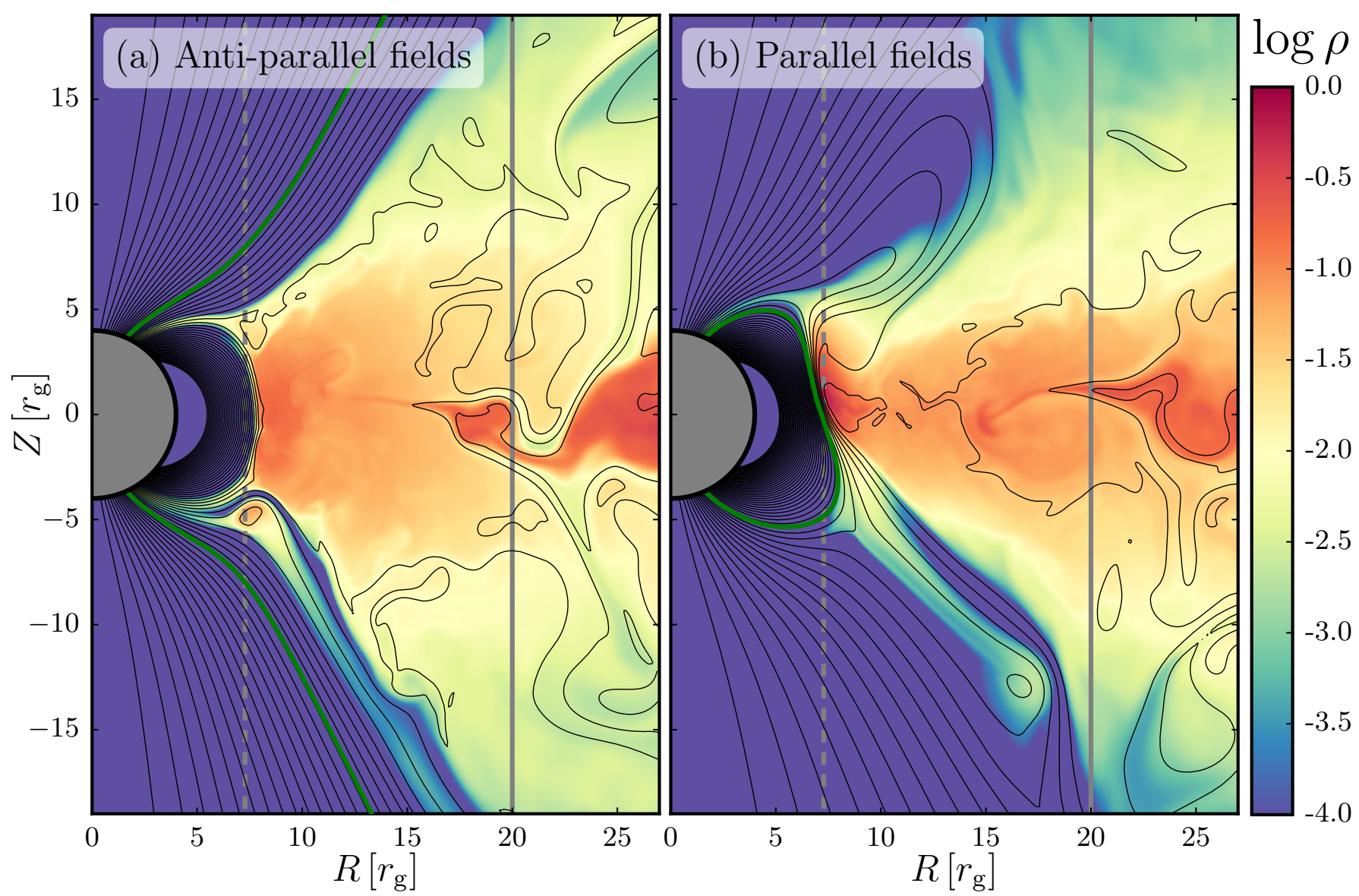

Figure 3. Effect of relative orientation of the stellar dipole and the initial magnetic field in the torus; $\mu=10$ and $t=21,760 r_{\mathrm{g}} / c$ in both cases. Markings are as in Figure 2. (a) Anti-parallel fields: the torus's field lines can directly reconnect with those of the stellar closed zone, facilitating accretion and increasing the open magnetic flux. (b) Parallel fields: direct reconnection is not possible, reducing the number of open field lines and suppressing accretion.

The bottom panel of Figure 5 shows the stellar torque separated into three contributions: the electromagnetic torque in the force-free $(\mathcal{F}>0.5)$ and $\operatorname{MHD}(\mathcal{F}<0.5)$ regions, and the material (or hydrodynamic) torque. The material torque is negligible in every case; by the time the accreting gas reaches the surface it is nearly in corotation with the star, having transferred its angular momentum to the stellar magnetic field whose stress applies the torque (Romanova et al. 2002).

The electromagnetic torque in the force-free region is always negative (spin-down) or zero, while its average in the MHD region (i.e., in the accretion columns) is generally positive. The simulations cover the range in total torque from strong spin-down $\left(R_{\mathrm{LC}}=20 r_{\mathrm{g}}\right)$ to weak spin-up (non-rotating star), with the $R_{\mathrm{LC}}=60 r_{\mathrm{g}}$ system in approximate spin equilibrium.

\section{CONCLUSIONS}

We have presented a method of combining force-free and standard MHD behavior in a self-consistent simu- lation in curved spacetime, and used this approach to perform the first relativistic-MHD simulations of accretion onto rotating, magnetized neutron stars. The spin frequencies are in the millisecond range, and our initial conditions, in which all of the accreting gas originates from an equilibrium torus beyond the light cylinder, allow the direct simulation of the transition between isolated (radio pulsar) and accreting (X-ray pulsar) states. The energy-conserving MHD evolution produces a thick accretion flow, whose interaction with the stellar magnetosphere is relevant to hard-state AMXPs, Z sources, and the pulsing ULXs; recently Takahashi \& Ohsuga (2017) have described a radiation-GRMHD simulation of accretion onto a non-rotating star, also aimed at the ULXs.

Our method can be described in covariant language for general spacetimes and coordinates, and may be useful in various contexts involving both regions with significant hydrodynamic inertia and those that are very light and magnetically dominated, such as black-hole 


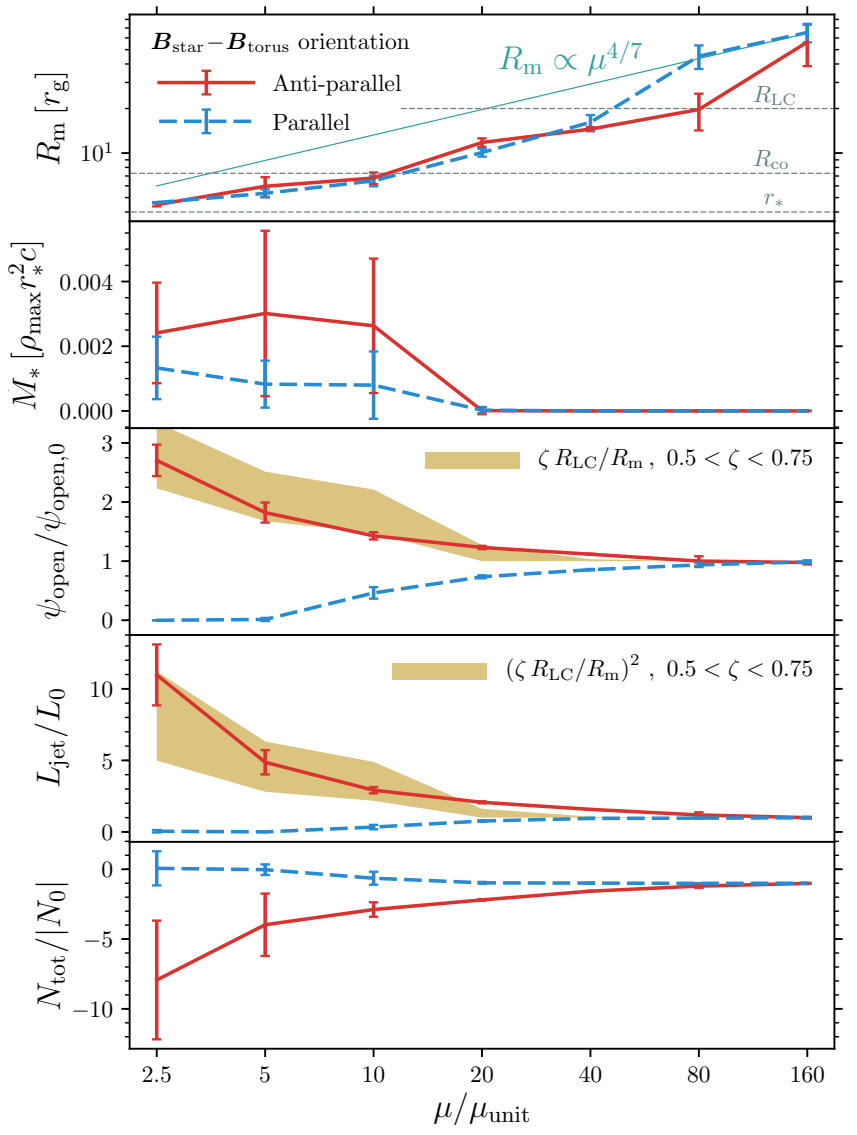

Figure 4. Averaged global quantities as a function of stellar magnetic moment, for $R_{\mathrm{LC}}=20 r_{\mathrm{g}}$. Vertical bars indicate the standard deviation over the averaging period. From top to bottom: magnetospheric radius; mass accretion rate onto the stellar surface; magnetic flux open through $r=R_{\mathrm{LC}}$ in the force-free region $(\mathcal{F}>0.5)$; total torque on the star measured at its surface; electromagnetic jet power. The shaded regions show predictions from a simple flux-opening model. Quantities subscripted with ' 0 ' indicate their measured isolated-state values; note that $N_{0}<0$ (i.e., causes spin-down).

jets, gamma-ray bursts, and compact-object mergers. It is straightforward to implement in existing codes, and adds only one new scalar variable and its associated advection equation to the MHD system.

By varying the star's magnetic field strength, four states of the star-disk system are produced, determined by the position of the disk-truncation radius with respect to the problem's other three characteristic locations: the stellar, corotation, and light-cylinder radii. These states include the three regimes previously explored with non-relativistic MHD simulations (boundary-layer and magnetically channeled accretion, and the propeller regime), together with a new state in which the pulsar's electromagnetic wind is power-

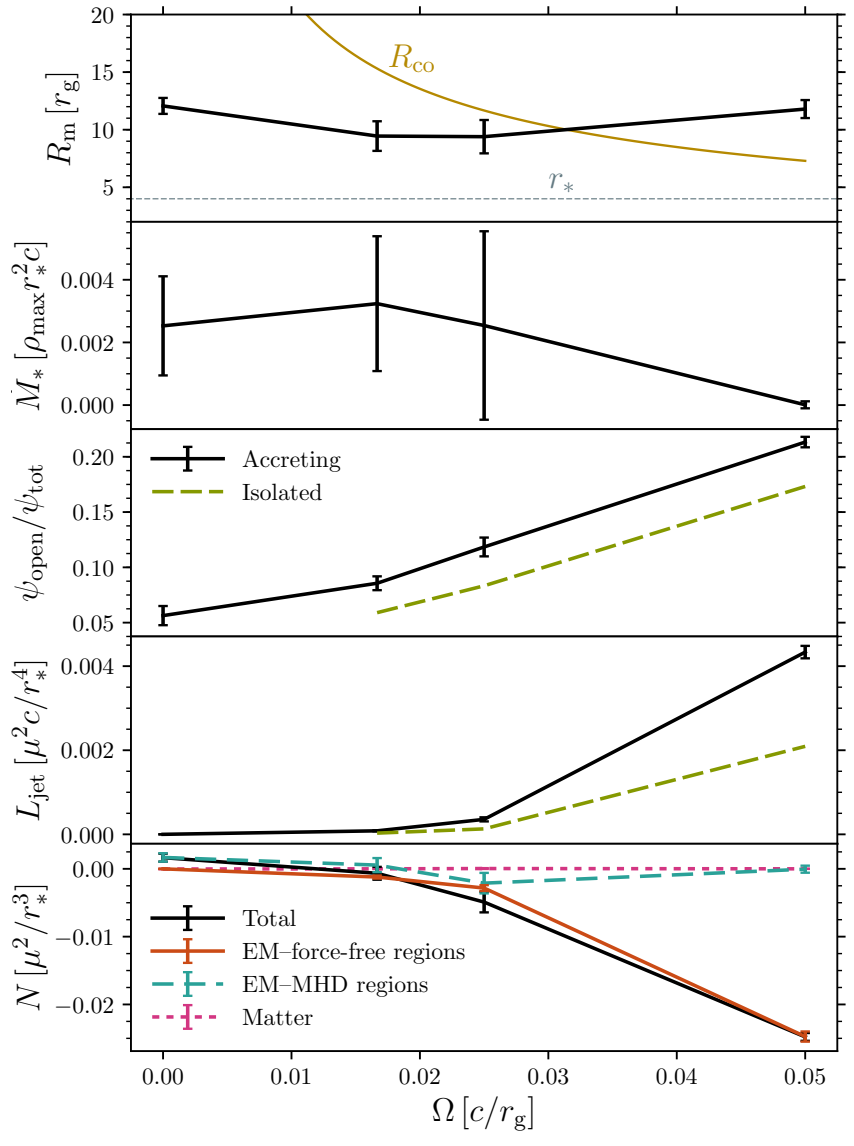

Figure 5. Averaged quantities as a function of spin frequency $\Omega=c / R_{\mathrm{LC}}$, for $\mu=20$ and the anti-parallel startorus field orientation. Panels are the same as in Figure 4, however, all are now plotted in absolute units; $\psi_{\text {tot }}$ is the total magnetic flux through the stellar surface. The dashed green lines indicate the open flux and electromagnetic wind power from an isolated pulsar.

ful enough to prevent the entry of the accretion flow through the light cylinder (e.g. Shvartsman 1970; Burderi et al. 2001). The pulsar wind drives trains of shocks and sound waves into the flow, possibly producing X-ray emission at large distances. We also find a new intermediate state, in which the accretion flow is usually inside the light cylinder, but is regularly expelled back through it by the star's rotating magnetic field - this expulsion changes the spin-down rate and potential for low-level stellar accretion, and may be related to the mode switching of the transitional millisecond pulsars (e.g. Linares et al. 2014).

In our axisymmetric simulations, the direction of the torus's magnetic loop significantly affects the system's evolution, an effect cleanly isolated by our deformeddipole initial conditions (see Section. 3). When the stellar and torus fields are anti-parallel, the accreting 
plasma efficiently opens the star's closed magnetic field, leading to a pair of powerful relativistic jets that are collimated by the thick accretion flow (Parfrey et al. 2016).

This mechanism may have operated in the neutronstar merger GW170817, whose unknown compact remnant produced a jet of power $\sim 10^{49}-10^{50} \mathrm{erg} \mathrm{s}^{-1}$ (Abbott et al. 2017; Margutti et al. 2017). The flux-opening jet model predicts $L_{\text {jet }} \sim 10^{52} B_{15}^{6 / 7} \dot{M}_{1}^{4 / 7} \nu_{1}^{2} \mathrm{erg} \mathrm{s}^{-1}$, where the stellar magnetic field strength, spin frequency, and accretion rate are normalized as $B_{15}=B_{*} / 10^{15} \mathrm{G}$, $\nu_{1}=\nu_{*} / 1 \mathrm{kHz}$, and $\dot{M}_{1}=\dot{M} / 1 \mathrm{M}_{\odot} \mathrm{s}^{-1}$. Similarly, our simulation with the largest ratio of jet power to isolated-pulsar-wind power has an average $L_{\text {jet }} \approx 11 L_{0}$ (see Fig. 4), giving $L_{\text {jet }} \approx 6.4 \times 10^{50} B_{15}^{2} \nu_{1}^{4} \mathrm{erg} \mathrm{s}^{-1}$. These estimates suggest that a rapidly rotating neutron star with a magnetar-strength field is a viable central engine for the observed short-duration gamma-ray burst. More speculatively, an early phase of rapid accretion may have caused the $1.7 \mathrm{~s}$ delay between gravitational waves and gamma-rays, as the accretion flow may have quenched the jet if it overwhelmed the magnetosphere, occurring if $R_{\mathrm{m}} \approx 3.5 B_{15}^{4 / 7} \dot{M}_{1}^{-2 / 7} \mathrm{~km} \ll r_{*}$ (Parfrey et al. 2016).

In the parallel-fields case, no opening occurs and jets are not produced, at least partly due to the limitations of axisymmetry, where a poloidal field loop cannot change its direction of circulation. This restriction is lifted in three-dimensional evolution, which is also necessary for creating a self-sustaining MRI dynamo and hence a longterm steady state. Though expected to be weaker in 3D, the magnitude of this effect on torques and jet powers seen in Figure 4 suggests that there may be interesting observational consequences to variations in any largescale net field advected inward by accretion.

KP was supported by NASA through Einstein Postdoctoral Fellowship grant number PF5-160142. AT was supported by the TAC fellowship and in part under grant no. NSF PHY-1125915. The simulations were performed on the SAVIO cluster provided by the Berkeley Research Computing program at the University of California, Berkeley, and on the Stampede cluster of the Texas Advanced Computing Center (TACC) of the University of Texas at Austin, via allocation AST150062.

\section{REFERENCES}

Abbott, B. P., et al. 2017, ApJL, 848, L12

Bachetti, M., Harrison, F. A., Walton, D. J., et al. 2014, Nature, 514, 202

Balbus, S. A., \& Hawley, J. F. 1991, ApJ, 376, 214

Belyaev, M. A., \& Parfrey, K. 2016, ApJ, 830, 119

Burderi, L., Possenti, A., D'Antona, F., et al. 2001, ApJL, 560, L71

Chakrabarti, S. K. 1985, ApJ, 288, 1

Contopoulos, I., Kazanas, D., \& Fendt, C. 1999, ApJ, 511, 351

De Villiers, J.-P., Hawley, J. F., \& Krolik, J. H. 2003, ApJ, 599, 1238

Fürst, F., Walton, D. J., Harrison, F. A., et al. 2016, ApJL, 831, L14

Gammie, C. F., McKinney, J. C., \& Tóth, G. 2003, ApJ, 589,444

Gruzinov, A. 2005, PhRvL, 94, 021101

Hasinger, G., \& van der Klis, M. 1989, A\&A, 225, 79

Hayashi, M. R., Shibata, K., \& Matsumoto, R. 1996, ApJL, 468, L37

Israel, G. L., Belfiore, A., Stella, L., et al. 2017, Science, 355,817

Kato, Y., Hayashi, M. R., \& Matsumoto, R. 2004, ApJ, 600, 338

Komissarov, S. S. 2006, MNRAS, 367, 19
Lii, P. S., Romanova, M. M., Ustyugova, G. V., Koldoba, A. V., \& Lovelace, R. V. E. 2014, MNRAS, 441, 86

Linares, M., Bahramian, A., Heinke, C., et al. 2014, MNRAS, 438, 251

Margutti, R., Berger, E., Fong, W., et al. 2017, ApJL, 848, L20

Michel, F. C. 1973, ApJL, 180, L133

Miller, K. A., \& Stone, J. M. 1997, ApJ, 489, 890

Noble, S. C., Gammie, C. F., McKinney, J. C., \& Del Zanna, L. 2006, ApJ, 641, 626

Papitto, A., Ferrigno, C., Bozzo, E., et al. 2013, Nature, 501,517

Parfrey, K., Beloborodov, A. M., \& Hui, L. 2012, MNRAS, 423, 1416

Parfrey, K., Spitkovsky, A., \& Beloborodov, A. M. 2016, ApJ, 822, 33

—. 2017, MNRAS, 469, 3656

Patruno, A., \& Watts, A. L. 2012, ArXiv e-prints, arXiv:1206.2727

Pringle, J. E., \& Rees, M. J. 1972, A\&A, 21, 1

Romanova, M. M., Ustyugova, G. V., Koldoba, A. V., \& Lovelace, R. V. E. 2002, ApJ, 578, 420

-. 2011, MNRAS, 416, 416

-. 2012, MNRAS, 421, 63

Shvartsman, V. F. 1970, Soviet Ast., 14, 527 
Spitkovsky, A. 2006, ApJL, 648, L51

Stappers, B. W., Archibald, A. M., Hessels, J. W. T., et al. 2014, ApJ, 790, 39

Takahashi, H. R., \& Ohsuga, K. 2017, ArXiv e-prints, arXiv:1707.07356
Tchekhovskoy, A., McKinney, J. C., \& Narayan, R. 2009, ApJ, 699, 1789

Tchekhovskoy, A., Spitkovsky, A., \& Li, J. G. 2013, MNRAS, 435, L1

van der Klis, M. 2000, ARA\&A, 38, 717

Wasserman, I., \& Shapiro, S. L. 1983, ApJ, 265, 1036

Zanni, C., \& Ferreira, J. 2009, A\&A, 508, 1117 